\title{
Segmentation in Echocardiographic Sequences Using Shape-Based Snake Model
}

\author{
Chen Sheng ${ }^{1}$, Yang Xin ${ }^{1}$, Yao Liping ${ }^{2}$, and Sun Kun ${ }^{2}$ \\ ${ }^{1}$ Institution of Image Processing and Pattern Recognition, \\ Shanghai Jiaotong University, Shanghai, P.R. China \\ chnshn@hotmail.com, yangxinasjtu.edu.cn \\ ${ }^{2}$ Shanghai Children's Medical Center, Shanghai Second Medical University, \\ Shanghai, P.R. China
}

\begin{abstract}
A novel method for segmentation of cardiac structures in temporal echocardiographic sequences based on the snake model is presented. The method is motivated by the observation that the structures of neighboring frames have consistent locations and shapes that aid in segmentation. To cooperate with the constraining information provided by the neighboring frames, we combine the template matching with the conventional snake model. Furthermore, in order to auto or semi-automatically segment the sequent images without manually drawing the initial contours in each image, generalized Hough transformation (GHT) is used to roughly estimate the initial contour by transforming the neighboring prior shape. As a result, the active contour can easily detect the desirable boundaries in ultrasound images.
\end{abstract}

\section{Introduction}

Endocardial boundary detection in ultrasound images is a necessary step to obtain both qualitative measurements (i.e., the detection of pathological deformation) and quantitative measurements (i.e., area, volume and etc.). Unfortunately, this is a difficult task due to the poor spatial and contrast resolutions, a high level of speckle noise and etc. To over come these problems, various algorithms are proposed to extract the boundaries of the region of interest (ROI) in echocardiographic images [1]. These approaches can be mainly categorized based on Markov random field [2], artificial neural network [3], mathematical morphological [4] and deformable model [5], etc. In these schemes, the deformable model [6], which is also known as the snake model, is the most important and popular model for noisy and low contrast image segmentation. In this paper, the main reason for using the snake model is that it allows the incorporation of geometric constraints.

However, the conventional deformable models have some deficiencies for boundary detection in ultrasound images. Firstly, the initial contour generally has to be placed quite close to the desirable boundary. Second, when the snake model is used to track the object in an image sequence by using the final contour from the previous frame as the initial contour in the current frame, the tracking works well only for small frame-to-frame displacement of ROI. Otherwise, the derived contour may be 
easily trapped in a local minimum formed by the noise. To remedy this problem, many techniques were proposed, for example, gradient vector flow (GVF) [7], dual snake [8] and discrete snake [9]. In this paper, it is noticed that the boundaries of any two adjacent images in a sequence are correlated to a certain degree. The result found in one image can be used as the shape template for the adjacent one. Thus, the only one rough shape template in a sequence needs to be given manually at the first step. For large frame-to-frame displacement of ROI, such as the mitral valve, GHT [10] is utilized to transform the shape template to an initial contour in the ROI. It has been proven that GHT is able to detect any arbitrary shape undergoing a geometric transformation in an image. Moreover, it has shown to be robust and can even be successfully used to detect overlapping or semi-occluded objects in noisy images.

Our method is based on the template matching which incorporates the prior shape template from the adjacent frame into the snake model. Optimizing the deformation energy between the shape template and the active contour, the shape of the active contour is constrained to be similar to the template in global while still allowing slight deformation locally. Under this energy criterion, the contour can escape from the local minimum caused by the speckle, the tissue-related textures.

\section{Methods}

Let $\Omega$ be a bounded open subset of $R^{2}$. Let $u_{0}: \bar{\Omega} \rightarrow R$ be a given image, and $C(s)=(x(s), y(s))(s \in[0,1])$ be a parameterized contour with $s$ the parameter of length. The shape-based snake model is to minimize the following energy:

$$
E\left(u_{0}, C_{d}, C_{t}\right)=\alpha E_{\text {int }}\left(C_{d}\right)+\beta E_{e x t}\left(u_{0}, C_{d}\right)+\eta E_{c o n}\left(C_{d}, C_{t}\right)
$$

where $C_{d}$ is the active contour, $C_{t}$ is the shape template.

$E_{\text {int }}\left(C_{d}\right)$ is the internal energy that controls the smoothness of the contour:

$$
E_{\text {int }}\left(C_{d}\right)=\int_{0}^{1}\left|C_{d}^{\prime}(s)\right|^{2} d s+\int_{0}^{1}\left|C^{\prime \prime}{ }_{d}(s)\right| d s
$$

$E_{\text {ext }}\left(u_{0}, C_{d}\right)$ is the external energy that attracts of the active contour evolving to the boundary of object. In this paper, it is calculated from the texture information instead of the local gradient in the ultrasound image. Furthermore, a blurring Gaussian filter is applied for better result. However, the blurred texture feature probably loses some object boundary information. Hence, the original image feature is also used to retain the boundary information. Let $T(x, y)$ denote the texture image after applying the texture analysis to the original image $u_{0}(x, y)$. The blurring Gaussian filter is applied to the texture image $T(x, y)$ to obtain the blurred texture image $T_{G}(x, y)$. Now the external energy $E_{e x t}$ is defined as:

$$
E_{e x t}\left(u_{0}, C_{d}\right)=-\left|\nabla u_{0}\left(C_{d}(s)\right)\right|-\left|\nabla T_{G}\left(C_{d}(s)\right)\right|
$$


$E_{c o n}\left(C_{d}, C_{t}\right)$ is the energy to measure the similarity between the active contour and the shape template. In this paper, our method has been inspired by the approach due to Duncan [11], who proposed a scheme for matching two contours based on the minimization of a quadratic fitting criterion, which consists of a curvature dependent bending energy term and a smoothness term.

Duncan [11] introduces a local bending energy measure of the form:

$$
E_{\text {curvature }}=\int\left(k_{C_{d}}\left(s^{\prime}\right)-k_{C_{t}}(s)\right)^{2} d s
$$

where $k_{C_{d}}\left(s^{\prime}\right)$ is the curvature of the active contour $C_{d}$ at $s^{\prime}$ as well as $k_{C_{t}}(s)$.

We also wish the displacement vector field to vary smoothly along the active contour:

$$
E_{\text {smooth }}=\int\left\|\frac{\partial\left(C_{d}\left(s^{\prime}\right)-C_{t}(s)\right)}{\partial s}\right\| d s
$$

So the criterion is composed of the curvature constraint and the smooth constraint:

$$
E_{\text {elastic }}=E_{\text {curvature }}+\lambda E_{\text {smooth }}
$$

\section{Initialization of the Active Contour}

The shape template must be approximated as a vector containing a sequential discrete points in order to solve by numerical method, $U=\left[u_{1}, u_{2}, \cdots, u_{n}\right]$, where $u_{i}=\left(u_{i x}, u_{i y}\right) \in\{(x, y): x, y=1,2, \cdots, M\}$. The same method is used for the active contour, $V=\left[v_{1}, v_{2}, \cdots, v_{n}\right]$.

Before processing the boundary detection by the snake, an initial contour must be draw. The purpose of the initialization is to place the initial contour as close as possible to the boundary in ROI in order to obtain a fast convergence in the boundary detection. In this paper, the GHT is applied to solve this problem. Let us define a geometric transformation of the shape template by:

$$
V=A U+t=\left[\begin{array}{ll}
a_{A} & b_{A} \\
c_{A} & d_{A}
\end{array}\right] \cdot\left[\begin{array}{l}
U_{x} \\
U_{y}
\end{array}\right]+\left[\begin{array}{c}
t_{x} \\
t_{y}
\end{array}\right] \quad\left(a_{A} d_{A}-b_{A} c_{A} \neq 0\right)
$$

where $A$ and $t$ correspond to a linear transformation and to a translation vector respectively. The potential location of the position parameters $t$ for the potential parameters $A$ of the linear transformation can be expressed as $t(U, V, A)=V-A U$. This method traces an initial contour in the parameter space, and after gathering all evidences for all ROI pixels, the maximum of the accumulator array defines the best values $A^{*}$ and $t^{*}$ which correspond to the transformation that maps the shape template to the echocardiographic image. The GHT can deliver a reliable estimation of the ROI position or a coarse initial contour. 


\section{Experiments and Results}

In this section, several examples are presented to illustrate the efficiency of the shapebased snake model for boundary detection in echocardiographic sequences. Six sequent ultrasound images with size $180 \times 180$ pixels were obtained from the Philip 5500 system, each covering one complete cardiac cycle and containing $F=16$ frames. The algorithm has been implemented using an Intel Pentium IV 2.4Ghz with 1 GB RAM, under the Visual C++ 6.0 environment.

To assess the performance of our segmentation method, we compared automatically detected cardio structure boundaries with the manual outlines. In this paper, four sets of manual outlines are given for each of the sequences.

Two sets of parameters are employed: the mean, the standard deviation (SD), and the maximum of the minimal distances from the derived boundary points to the manual outline. They are used to measure the difference between the derived contour and the outline in one frame of a sequence.

Let $C_{d}$ and $C_{m}$ denote the derived contour and the manual outline, respectively.

$\sim$ For each $p_{i} \in C_{d}$, find $p_{i}^{*} \in C_{m}$ so that $p_{i}^{*}=\arg \min _{\forall p_{j}^{*} \in C_{m}}\left\|p_{i}-p_{j}^{*}\right\|$, where $\left\|p_{i}-p_{j}^{*}\right\|$ means the Euclidean distance between the two pixels.

$\sim$ For all $\left(p_{i}, p_{i}^{*}\right)$, computer the Euclidean distance $d$.

$\sim$ Compute the mean, the SD and the maximum of $\left\{d \mid \forall p_{i} \in C_{d}\right\}$.

We need another set of parameters to evaluate the segmentation results for the whole sequence, so the mean and the SD of the mean absolute distance (MAD) are defined as follow:

$\sim$ The MAD between two contours $A$ and $B$ is defined as: $D(A, B)=\frac{1}{2}\left\{\frac{1}{n} \sum_{i=1}^{n} d\left(a_{i}, B\right)+\frac{1}{m} \sum_{i=1}^{m} d\left(b_{i}, A\right)\right\}$.

$\sim$ Compute the mean, the SD of $\{D \mid \forall D \in S\}$, where $S$ is all the MADs need to be calculated for a sequence.

\subsection{Process of Segmentation}

Fig. 1 shows the segmentation process for a mitral valve sequence. The initial contour of the $(k+1)$ th image obtained directly from the final contour of the $k$ th image is shown in Fig.1 (c). Fig.1 (b) presents the initial contour, which has been transformed by GHT. In the Fig.1 (d), we can see that the segmentation result rather coincides with the contour manually defined by an independent doctor in Fig.1 (e) when using GHT to locate the initial contour. On the other hand, we can see that the shape-based snake model treats well when there is a gap in the tip of the leaflet under the shape constraint. 


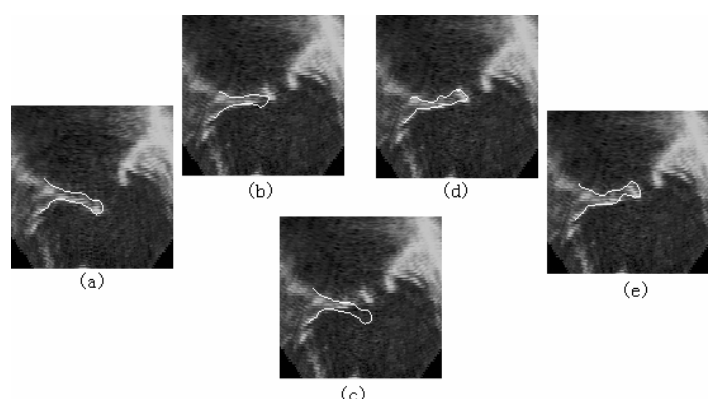

Fig. 1. Example of segmentation for mitral valve; (a) the kth image with finial contour; (b) the $(\mathrm{k}+1)$ th image with initial contour from the kth image using GHT; $(\mathrm{c})$ the $(\mathrm{k}+1)$ th image with initial contour direct from the kth image; $(\mathrm{d})$ the $(\mathrm{k}+1)$ th image with segmentation result using initial contour in (b); (e) manual outline for the $(k+1)$ th image

Table 1. The mean, the SD and the maximum of the minimal distances for Fig.2

\begin{tabular}{llll}
\hline Minimal distances & Mean [pixels] & SD [pixels] & Max [pixels] \\
\hline Using GHT & 1.5 & 1.06 & 5.2 \\
Without using GHT & 1.4 & 1.15 & 5.1 \\
\hline
\end{tabular}

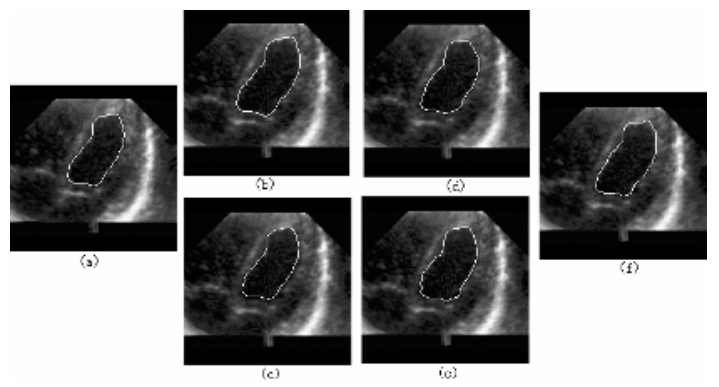

Fig. 2. Example of segmentation for left ventricle; (a) the kth image with finial contour; (b) the $(\mathrm{k}+1)$ th image with initial contour from the kth image using GHT; $(\mathrm{c})$ the $(\mathrm{k}+1)$ th image with initial contour direct from the kth image; $(\mathrm{d})$ the $(\mathrm{k}+1)$ th image with segmentation result using initial contour in (b); (e) the (k+1)th image with segmentation result using initial contour in (c); (f) manual outline for the $(\mathrm{k}+1)$ th image

It may be reasonable to say that the segmentation result closely follows the desired boundary. Nevertheless, the algorithm fails when using the initial contour in Fig.1 (c) although the same energy weighting factors ( $\alpha=1.0, \beta=1.0, \eta=0.5$ ) are given.

GHT is not needed in all situations such as the small frame-to-frame displacement of the structure. Fig.3 (e) shows the segmentation result for the left ventricle with the initial contour direct from the previous image is identical to that using GHT to locate the initial contour (Fig.3 (d)). The evaluated parameters of the segmentation results are shown in Table 1. Both the mean and the SD of the minimal distances are near to each other. 


\subsection{Segmentation of Endocaridal Boundaries in Sequences}

In four sequences, the algorithm was used to segment the endocaridal boundaries. Some frames from the first sequence are shown in Fig.3.

Table 2 shows the mean and the SD of the MADs for the whole sequence between the algorithm-generated contours and the four sets of manual outlines and between different manual outlines. These experiments show that the segmentation results compare well to the manual outlines for the endocaridal boundaries.
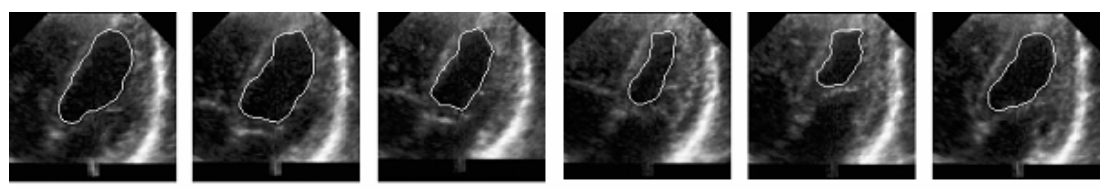

Fig. 3. Characteristic frames showing the segmentation results of the left ventricle

Table 2. Results of the comparison between the algorithm-generated contours and the manual outlines

\begin{tabular}{ccccc}
\hline & Seq1 & Seq2 & Seq3 & Seq4 \\
\hline Mean of MADs between snake and outlines [pixels] & 1.22 & 1.75 & 1.61 & 1.18 \\
SD of MADs between snake and outlines [pixels] & 0.26 & 0.35 & 0.45 & 0.31 \\
Mean of MADs between different manual outlines [pixels] & 1.32 & 1.65 & 1.41 & 1.24 \\
SD of MADs between different manual outlines [pixels] & 0.22 & 0.34 & 0.30 & 0.28 \\
\hline
\end{tabular}

\subsection{Segmentation of Mitral Valve Sequences}

The algorithm performance was evaluated on two sequences of long axis view images of the mitral valve. Characteristic frames from the first sequence are shown in Fig.4. As one could expect, the differences of ROI between any two adjacent frames are larger, but the algorithm performance is still comparable to the manual segmentations.
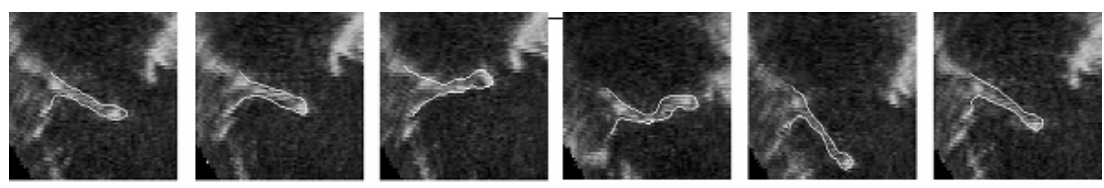

Fig. 4. Characteristic frames showing the segmentation results of the mitral valve

Table 3. Results of the comparison between the algorithm-generated contours and the manual outlines for sequences containing images of the mitral valve

\begin{tabular}{lll}
\hline & Seq1 & Seq2 \\
\hline Mean of MADs between snake and outlines [pixels] & 2.14 & 2.02 \\
SD of MADs between snake and outlines [pixels] & 0.71 & 0.56 \\
Mean of MADs between different manual outlines [pixels] & 1.81 & 1.63 \\
SD of MADs between different manual outlines [pixels] & 0.69 & 0.52 \\
\hline
\end{tabular}


Table 3 shows the evaluated results for mitral valve sequences. In this Table, we can see that the mean and the SD are larger than those in Table 2. It may be ascribed to at least two factors. The first one is that the manual outlines may vary with experts. The second factor is that the contours in the mitral valve images are open. The starting points and the ending points defined by the experts may vary largely. As a result, the MAD between the open contours may be larger than that between closed contours.

\subsection{Determination of Weighting Factors}

In our experiments, the weighting factors $\alpha, \beta, \eta$ are set in Table 4 . The motion of the mitral valve is very irregular, frame-to-frame displacements are several times larger than the leaflet thickness. At those phases, the leaflet rotates, translates and deforms at the same time. As a result, the difference of shape between two adjacent images may be large.

Table 4. Values of parameters used in the algorithm

\begin{tabular}{lccc}
\hline & $\alpha$ & $\beta$ & $\eta$ \\
\hline endocaridal sequences & 1.0 & 1.0 & 2.0 \\
Mitral valve sequences & 1.0 & 1.0 & 0.5 \\
\hline
\end{tabular}

GHT algorithms are known to be computationally expensive (about 6 min for a sequence in our experiments) and they are not needed in all situations. So, in our method, the GHT was separated from the snake deformation process. A user can intervene when or where GHT to be used. However, these algorithms do not need user's supervision during the segmentation process. The user's interaction was needed in just one frame for a sequence.

\section{Conclusions}

In this paper, an innovative model has been proposed for echocardiographic image segmentation, namely, the shape-based snake model. The proposed shape-based model aims to incorporate the template matching and the GHT with the snake model. The model can resist the speckle noise, tissue-related textures and artefacts, and guide the active contour deform to the desirable boundary. The principal idea of this model is to use GHT to estimate the initial contour, and then using the elastic deformation energy between the shape template and the active contour to guide the contour deform from the local minimum. Our method does not need to draw a precise shape template, but rather a rough contour regardless of its position, scaling and rotation only once in a sequence.

\section{Acknowledgments}

This work was partially supported by National Science Research Program of China (No. 2004BA714802) and Shanghai Science and Technology Development Foundation (034119820). 


\section{References}

1. Sher DB, Revankar S. Computer methods in quantitation of cardiac wall parameters from two-dimensional echocardiograms: A survey. Int. J. Cardiac Imaging 1992;8;11-26.

2. Dias JMB, Leitao JMN. Wall position and thickness estimation from sequence of echocardiographic images. IEEE Trans on Medical Imaging 1996;15;25-38.

3. Kotropolulos C. Nonlinear ultrasonic image processing based on signak-adaptive filters and self-organizing neural networks. IEEE Trans Image Processing 1994;3;65-77.

4. Thomas JG, Peters RA, Jeanty P. Automatic segmentation of ultrasound images using morphological operators. IEEE Trans Medical Imaging 1991;10;180-186.

5. Hass C, Ermert H, Holt S. Segmentation of 3-D intravascular ultrasonic images based on a random field model. Ultrasound Med Biol 2000;26;297-306.

6. Kass M, Witkin A, Terzopoulos D. Snakes: active contour models. International Journal of Computer Vision 1987;1;321-331.

7. Xu C, Prince JL. Snakes, shapes, and gradient vector flow. IEEE Trans Image Processing 1998;7;359-369.

8. Gunn SR, Nixon MS. A robust snake implementation: A dual active contour. IEEE Trans PAMI 1997;19;63-68.

9. Lobregt S, Viergever MA. A discrete active contour model. IEEE Trans Medical Imaging $1995 ; 14 ; 12-24$.

10. 10.Ballard DH. Generalizing the Hough transform to detect arbitrary shapes. Pattern Recognition 1981;13;111-122.

11. 11.Duncan JS, Owen R. Shape-based tracking of left ventricular wall motion. Computers in Cardiology 1990. IEEE Computer Society, Chicago, Illinois. 1990 September;23-26. 